\title{
Evaluation of Bio-pesticides against Fall Armyworm, Spodoptera frugiperda (J. E. Smith) in Maize
}

\author{
C. B. Dhobi ${ }^{1 *}$, M. B. Zala ${ }^{2}$, H. S. Verma ${ }^{3}$, D. B. Sisodiya ${ }^{1}$, R. K. Thumar ${ }^{4}$,

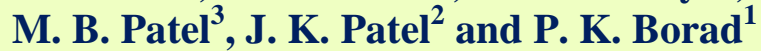 \\ ${ }^{1}$ Department of Entomology, B. A. College of Agriculture, Anand Agricultural University, \\ Anand 388 110, Gujarat, India \\ ${ }^{2} A R S$, Sansoli, $A A U$, Anand, India \\ ${ }^{3} \mathrm{MMRS}$, Godhra, AAU, Anand, India \\ *Corresponding author
}

\section{A B S T R A C T}

\begin{tabular}{|l|}
\hline K e y w o r d s \\
Fall armyworm, \\
Bio-pesticides, \\
Invasive pest, \\
Maize, Yield \\
\hline Article Info \\
\hline $\begin{array}{l}\text { Accepted: } \\
\text { 15 July 2020 } \\
\text { Available Online: } \\
\text { 10 August } 2020\end{array}$ \\
\hline
\end{tabular}

Introduction

Maize is an emerging third most important cereal crop after rice and wheat in India. Andhra Pradesh, Tamil Nadu, Rajasthan, Maharashtra, Bihar, Uttar Pradesh, Madhya Pradesh and Gujarat account for 85 per cent of India's maize production. Recently, the occurrence of a new invasive exotic pest Spodoptera frugiperda (J.E. Smith), a lepidopteron insect has been suspected on maize crop in Karnataka (Shylesha et al.,
An experiment was conducted under field condition at three locations viz., Entomology Farm, B. A. College of Agriculture, AAU, Anand, Agricultural Research Station, Sansoli and main maize research Station, Godhra during Kharif, 2019 to determine the efficacy of different biopesticides against the fall armyworm, Spodoptera frugiperda in maize (GAYMH-1) by using Randomized Block Design (RBD) with three replications. The lowest larval population (1.81 larvae /10 plants and 2.03 larvae /10 plants), minimum plant damage (15.34\% and $17.70 \%)$ and cob damage $(15.19 \%$ and $15.19 \%)$ was observed in the plot treated with Nomuraea rileyi 1\% WP @ 40 g/10 lit water and it was at par with Bacillus thuringiensis var.kurstaki $1 \%$ WG @ 20 g/10 lit water, respectively. Of the tested biopesticides, the highest grain and fodder yield was recorded from the plot treated with N. rileyi 1\% WP (2957 and $4069 \mathrm{~kg} / \mathrm{ha}$ ) and followed by B. thuringiensis (2932 and 4033 $\mathrm{kg} / \mathrm{ha})$. 
and sometimes this can lead to extensive defoliation and a reduction in the growth potential of the plant. In conditions of heavy infestations, the caterpillar sometimes burrows into the corn ear through the husk and feeds on the kernel and this damage the quality of the corn. The densities of caterpillar finally reduced to one or two caterpillar per plant due to their cannibalistic behaviour (Capinera, 2008). Sisodiya et al., (2018) reported the occurrence of invasive pest, fall armyworm, S. frugiperda in the maize field of Anklav taluka of Anand district of Gujarat. As $S$. frugiperda is polyphagous pest, as per the available literature, efforts are needed to manage the pest and to check its further spread and to attack other crops. For this purpose, insecticides are the main method to control $S$. frugiperda in corn in Brazil, however, it pollutes the environment when they are used indiscriminately. Another option is the biological control with several beneficial organisms acting as natural enemies, viz., parasitoids, predators, fungi, virus, bacteria and nematodes (Cruz et al., 2002). Hence, the present experiment was conducted to evaluate the effectiveness of some biopesticides for controlling FAW in maize.

\section{Materials and Methods}

In order to study the evaluation of different biopesticides against the fall armyworm, $S$. frugiperda in maize, a field experiment was carried out during Kharif, 2019 at three locations viz., Entomology Farm, B. A. College of Agriculture, AAU, Anand, Agricultural Research Station, Sansoli and main maize research Station, Godhra in Randomized Block Design (RBD) with 10 treatments and 3 replications each having plot size of 6.0 x $3.6 \mathrm{~m}$. Maize variety GAYMH-1 was sown at spacing of $60 \times 20 \mathrm{~cm}$ on $15^{\text {th }}$, $22^{\text {nd }}$ and $18^{\text {th }}$ July, 2019 in different locations respectively. Maize crop was raised by following standard agronomical practices except pest control measures. Treatments were used: $\mathrm{T}_{1}$ Beauveria bassiana $5 \% \mathrm{WP}$ $\left(1 \times 10^{9} \mathrm{cfu} / \mathrm{g}\right), \quad \mathrm{T}_{2}$ Metarhizium anisopliae $1.15 \%$ WP $\left(1 \times 10^{9} \mathrm{cfu} / \mathrm{g}\right), \mathrm{T}_{3}$ Bacillus thuringiensis $1 \% \mathrm{WG}, \mathrm{T}_{4}$ Nomuraea rileyi $1 \%$ WP $\left(2 \times 10^{8} \mathrm{cfu} / \mathrm{g}\right), \mathrm{T}_{5}$ Azadirachtin 1500 ppm, $\mathrm{T}_{6}$ Neem seed kernel extracts $5 \%, \mathrm{~T}_{7}$ Tobacco decoction $2 \%$ (cold method), $\mathrm{T}_{8}$ Lantana camara leaf water extracts $10 \%, \mathrm{~T}_{9}$ Green Chilli (chilli variety GVC-111) water extracts $10 \%$ and $\mathrm{T}_{10}$ control. In the entire treatments sticker was added @ $0.15 \%$. The first spray was made at initiation of pest. The second and third sprays were applied after 10 days interval of first spray. Spray fluid was applied to the extent of slight run off using knapsack sprayer. The number of larva(e) and damaged plants were counted from randomly selected ten plants before as well as 5 and 10 days after each application. Numbers of damaged cobs were recorded at harvest. The grain and fodder yield were also recorded from each net plot and converted into $\mathrm{kg} / \mathrm{ha}$. The data obtained thus were, subjected to statistical analysis after appropriate transformation to draw valid conclusion.

\section{Results and Discussion}

The data on pooled over periods, sprays and locations of fall armyworm during 2019 are presented in Table 1 to 5 , respectively. The efficacy of different biopesticides is adjudged based on pooled over periods.

\section{Larval population (No. of larvae/10 plants)}

The data on larval population pooled over three locations before spraying of biopesticides showed non significant differences which indicated homogeneous distribution of pest in the experimental plots at all locations i.e., Anand, Sansoli and Godhra (Table 1 and 3). All the biopesticides treatments were found significantly superior 
to control till 10 days of application in all the three sprays, pooled over periods as well as pooled over periods and sprays.

The data on pooled over periods of first spray differed significantly to each other. The lowest population of fall armyworm was recorded in plots treated with Nomuraea rileyi 1\% WP (2.19 larvae/10 plants) and it was at par with Bacillus thuringiensis $1 \%$ WG (2.32 larvae/10 plants). These two treatments significantly superior to rest of the biopesticides.

The treatment of azadirachtin 1500 ppm (2.78 larvae/10 plants) and Beauveria bassiana 5\% WP (2.81 larvae/10 plants) remained next effective in controlling the pest. Remaining bio-pesticides performed equally against fall armyworm in maize. The Lantana camara leaf water extract recorded highest larval population (3.54 larvae/10 plants).

The data on pooled over periods of second spray revealed that Nomuraea rileyi $1 \% \mathrm{WP}$ (1.84 larvae /10 plants) recorded the lowest larval population and it was at par with $B$. thuringiensis $1 \% \mathrm{WG}$ (2.03 larvae/10 plants). Azadirachtin 1500 ppm (2.32 larvae/10 plants) and $B$. bassiana 5\% WP (2.42 larvae/10 plants) registered next best effective treatments in controlling the pest. Of the tested biopesticides, green chilli water extracts found least effective by recording the highest larval population (3.83 larvae /10 plants) and it was at par with $L$. camara leaf water extract (3.66 larvae /10 plants). More or less similar trend of effectiveness was observed in pooled over periods of third spray as noticed after pooled over periods of second spray.

Overall, the data on pooled over periods, sprays and locations showed the lowest larval population in $N$. rileyi $1 \%$ WP (1.81 larvae $/ 10$ plants) and it was at par with $B$. thuringiensis $1 \%$ WG (2.03 larvae /10 plants). B. bassiana 5\% WP (2.42 larvae/10 plants) and Azadirachtin 1500 ppm (2.46 larvae /10 plants) were at par and stood second in position.

Remaining bio-pesticides perform equally against fall armyworm in maize. The green chilli water extracts recorded the highest population (3.83 larvae /10 plants) and it was at par with the L. camara leaf water extract (3.66 larvae /10 plants) in maize.

\section{Plant damage (\%)}

The data on plant damage pooled over three locations before spraying of biopesticides showed non significant differences which indicated homogeneous distribution of pest in the experimental plots at three locations i.e., Anand, Sansoli and Godhra (Table 2 and 3). All the biopesticides treatments were found significantly superior to control till 10 days of application in all the three sprays, pooled over periods as well as pooled over periods and sprays.

The data on pooled over periods of first spray differed significantly to each other. Minimum plant damage caused by fall armyworm was found in plots treated with $N$. rileyi $1 \%$ WP $(18.40 \%)$ and it was at par with $B$. thuringiensis $1 \% \mathrm{WG}(20.27 \%)$.

These two treatments were found significantly superior to rest of the bio-pesticides. $B$. bassiana 5\% WP (24.89\%), azadirachtin 1500 ppm $(25.09 \%)$ and neem seed kernel extracts $(26.51 \%)$ were found statically at par in reducing the maize plant damage due to fall armyworm.

The green chilli water extracts recorded maximum (32.87\%) plant damage and it was at par with $L$. camara leaf water extracts $(32.58 \%)$. 
Table.1 Evaluation of bio-pesticides against fall armyworm, S. frugiperda infesting maize (Pooled over periods, sprays and locations)

\begin{tabular}{|c|c|c|c|c|c|c|c|c|c|c|c|c|}
\hline \multirow{3}{*}{$\begin{array}{l}\text { Tr. } \\
\text { No. }\end{array}$} & \multirow[t]{3}{*}{ Treatments } & \multicolumn{11}{|c|}{ No. of larva(e) /10 plants days after spray } \\
\hline & & \multirow{2}{*}{$\begin{array}{l}\text { Before } \\
\text { spray }\end{array}$} & \multicolumn{3}{|c|}{ First } & \multicolumn{3}{|c|}{ Second } & \multicolumn{3}{|c|}{ Third } & \multirow{2}{*}{$\begin{array}{l}\text { Pooled over } \\
\text { periods and } \\
\text { sprays }\end{array}$} \\
\hline & & & 5 & 10 & Pooled & 5 & 10 & Pooled & 5 & 10 & Pooled & \\
\hline 1 & $\begin{array}{l}\text { Beauveria bassiana } 5 \% \mathrm{WP} \\
(40 \mathrm{~g} / 10 \text { lit water) }\end{array}$ & $\begin{array}{c}1.91 \\
(3.15)\end{array}$ & $\begin{array}{l}1.69^{\mathrm{ab}} \\
(2.36)\end{array}$ & $\begin{array}{l}1.94^{\mathrm{ab}} \\
(3.26)\end{array}$ & $\begin{array}{l}1.82^{\mathrm{bc}} \\
(2.81)\end{array}$ & $\begin{array}{l}1.75^{\mathrm{ab}} \\
(2.56)\end{array}$ & $\begin{array}{l}1.66^{\mathrm{bc}} \\
(2.26)\end{array}$ & $\begin{array}{l}1.71^{\mathrm{bc}} \\
(2.42)\end{array}$ & $\begin{array}{l}1.55^{\mathrm{bc}} \\
(1.90)\end{array}$ & $\begin{array}{l}1.66^{\mathrm{bc}} \\
(2.26)\end{array}$ & $\begin{array}{l}1.61^{\mathrm{bc}} \\
(2.09)\end{array}$ & $\begin{array}{l}1.71^{\mathrm{b}} \\
(2.42)\end{array}$ \\
\hline 2 & $\begin{array}{l}\text { Metarhizium anisopliae } 1.15 \% \mathrm{WP} \\
\text { (40 g/10 lit water) }\end{array}$ & $\begin{array}{c}1.88 \\
(3.03)\end{array}$ & $\begin{array}{l}1.73^{\mathrm{abc}} \\
(2.49)\end{array}$ & $\begin{array}{l}1.99^{\mathrm{abc}} \\
(3.46)\end{array}$ & $\begin{array}{l}1.86^{\text {cd }} \\
(2.96)\end{array}$ & $\begin{array}{l}1.85^{\text {bcd }} \\
(2.92)\end{array}$ & $\begin{array}{l}1.76^{\mathrm{c}} \\
(2.60)\end{array}$ & $\begin{array}{l}1.80^{\text {cd }} \\
(2.78)\end{array}$ & $\begin{array}{l}1.70^{\mathrm{c}} \\
(2.39)\end{array}$ & $\begin{array}{l}1.80^{\mathrm{c}} \\
(2.74)\end{array}$ & $\begin{array}{l}1.76^{\mathrm{c}} \\
(2.60)\end{array}$ & $\begin{array}{l}1.81^{\mathrm{c}} \\
(2.78)\end{array}$ \\
\hline 3 & $\begin{array}{l}\text { Bacillus thuringiensis } 1 \% \mathrm{WG} \\
(20 \mathrm{~g} / 10 \text { lit water) }\end{array}$ & $\begin{array}{c}1.91 \\
(3.15)\end{array}$ & $\begin{array}{l}1.62^{\mathrm{a}} \\
(2.12)\end{array}$ & $\begin{array}{l}1.73^{\mathrm{a}} \\
(2.49)\end{array}$ & $\begin{array}{l}1.68^{\mathrm{ab}} \\
(2.32)\end{array}$ & $\begin{array}{l}1.58^{\mathrm{a}} \\
(2.00)\end{array}$ & $\begin{array}{l}1.59^{\mathrm{ab}} \\
(2.03)\end{array}$ & $\begin{array}{l}1.59^{\mathrm{ab}} \\
(2.03)\end{array}$ & $\begin{array}{l}1.48^{\mathrm{ab}} \\
(1.69)\end{array}$ & $\begin{array}{l}1.49^{\mathrm{ab}} \\
(1.72)\end{array}$ & $\begin{array}{l}1.49^{\mathrm{ab}} \\
(1.72)\end{array}$ & $\begin{array}{l}1.59^{\mathrm{a}} \\
(2.03)\end{array}$ \\
\hline 4 & $\begin{array}{l}\text { Nomuraea rileyi } 1 \% \mathrm{WP} \\
(40 \mathrm{~g} / 10 \text { lit water })\end{array}$ & $\begin{array}{c}1.91 \\
(3.15)\end{array}$ & $\begin{array}{l}1.58^{\mathrm{a}} \\
(2.00)\end{array}$ & $\begin{array}{l}1.69^{\mathrm{a}} \\
(2.36)\end{array}$ & $\begin{array}{l}1.64^{\mathrm{a}} \\
(2.19)\end{array}$ & $\begin{array}{l}1.58^{\mathrm{a}} \\
(2.00)\end{array}$ & $\begin{array}{r}1.47^{\mathrm{a}} \\
(1.66)\end{array}$ & $\begin{array}{l}1.52^{\mathrm{a}} \\
(1.84)\end{array}$ & $\begin{array}{r}1.37^{\mathrm{a}} \\
(1.38)\end{array}$ & $\begin{array}{l}1.39^{\mathrm{a}} \\
(1.43)\end{array}$ & $\begin{array}{r}1.39^{\mathrm{a}} \\
(1.43)\end{array}$ & $\begin{array}{r}1.52^{\mathrm{a}} \\
(1.81)\end{array}$ \\
\hline 5 & $\begin{array}{l}\text { Azadirachtin } 1500 \mathrm{ppm} \\
(40 \mathrm{ml} / 10 \text { lit water) }\end{array}$ & $\begin{array}{c}2.01 \\
(3.54)\end{array}$ & $\begin{array}{l}1.73^{\mathrm{abc}} \\
(2.49)\end{array}$ & $\begin{array}{l}1.88^{\mathrm{ab}} \\
(3.03)\end{array}$ & $\begin{array}{l}1.81^{\mathrm{bc}} \\
(2.78)\end{array}$ & $\begin{array}{l}1.72^{\mathrm{bc}} \\
(2.46)\end{array}$ & $\begin{array}{l}1.62^{\mathrm{bc}} \\
(2.12)\end{array}$ & $\begin{array}{l}1.67^{\mathrm{bc}} \\
(2.32)\end{array}$ & $\begin{array}{l}1.62^{\mathrm{bc}} \\
(2.12)\end{array}$ & $\begin{array}{l}1.72^{\mathrm{c}} \\
(2.46)\end{array}$ & $\begin{array}{l}1.67^{\mathrm{c}} \\
(2.29)\end{array}$ & $\begin{array}{l}1.72^{\mathrm{bc}} \\
(2.46)\end{array}$ \\
\hline 6 & $\begin{array}{l}\text { Neem seed kernel extract } 5 \% \\
(500 \mathrm{~g} / 10 \text { lit water) }\end{array}$ & $\begin{array}{c}1.79 \\
(2.70)\end{array}$ & $\begin{array}{l}1.77^{\mathrm{abc}} \\
(2.63)\end{array}$ & $\begin{array}{l}1.94^{\mathrm{ab}} \\
(3.26)\end{array}$ & $\begin{array}{l}1.86^{\mathrm{cd}} \\
(2.96)\end{array}$ & $\begin{array}{l}1.77^{\mathrm{bcd}} \\
(2.63)\end{array}$ & $\begin{array}{l}1.72^{\mathrm{bc}} \\
(2.46)\end{array}$ & $\begin{array}{l}1.75^{\mathrm{c}} \\
(2.56)\end{array}$ & $\begin{array}{l}1.65^{\mathrm{c}} \\
(2.22)\end{array}$ & $\begin{array}{l}1.72^{\mathrm{c}} \\
(2.46)\end{array}$ & $\begin{array}{l}1.69^{c} \\
(2.36)\end{array}$ & $\begin{array}{l}1.77^{\mathrm{bc}} \\
(2.63)\end{array}$ \\
\hline 7 & $\begin{array}{l}\text { Tobacco decoction } 2 \% \text { (cold method) } \\
\text { (200 g/10 lit water) }\end{array}$ & $\begin{array}{l}1.83 \\
(2.85)\end{array}$ & $\begin{array}{l}1.79^{\mathrm{d}} \\
(2.70)\end{array}$ & $\begin{array}{l}2.06^{\mathrm{bc}} \\
(3.74)\end{array}$ & $\begin{array}{l}1.92^{\text {cd }} \\
(3.19)\end{array}$ & $\begin{array}{l}1.88^{\text {cde }} \\
(3.03)\end{array}$ & $\begin{array}{l}1.97^{\mathrm{d}} \\
(3.38)\end{array}$ & $\begin{array}{l}1.93^{\mathrm{de}} \\
(3.22)\end{array}$ & $\begin{array}{l}1.88^{\mathrm{d}} \\
(3.03)\end{array}$ & $\begin{array}{l}2.03^{\mathrm{d}} \\
(3.62)\end{array}$ & $\begin{array}{l}1.96^{\mathrm{d}} \\
(2.34)\end{array}$ & $\begin{array}{l}1.94^{\mathrm{d}} \\
(3.26)\end{array}$ \\
\hline 8 & $\begin{array}{l}\text { Lantana camara leaf water extract } 10 \% \\
(1000 \mathrm{~g} / 10 \text { lit water) }\end{array}$ & $\begin{array}{l}1.85 \\
(2.92)\end{array}$ & $\begin{array}{l}1.89^{\text {cd }} \\
(3.07)\end{array}$ & $\begin{array}{l}2.13^{\text {cd }} \\
(4.04)\end{array}$ & $\begin{array}{l}2.01^{\mathrm{d}} \\
(3.54)\end{array}$ & $\begin{array}{l}1.99^{\mathrm{de}} \\
(3.46)\end{array}$ & $\begin{array}{l}2.08^{\mathrm{d}} \\
(3.83)\end{array}$ & $\begin{array}{l}2.03^{\mathrm{e}} \\
(3.66)\end{array}$ & $\begin{array}{l}1.99^{\mathrm{de}} \\
(3.46)\end{array}$ & $\begin{array}{r}2.12^{\mathrm{d}} \\
(3.99)\end{array}$ & $\begin{array}{l}2.06^{\mathrm{de}} \\
(3.74)\end{array}$ & $\begin{array}{l}2.04^{\mathrm{e}} \\
(3.66)\end{array}$ \\
\hline 9 & $\begin{array}{l}\text { Green chilli water extract } 10 \% \\
(1000 \mathrm{~g} / 10 \text { lit water })\end{array}$ & $\begin{array}{c}2.04 \\
(3.66)\end{array}$ & $\begin{array}{l}1.85^{\text {bcd }} \\
(2.92)\end{array}$ & $\begin{array}{l}2.08^{\mathrm{bc}} \\
(3.83)\end{array}$ & $\begin{array}{l}1.97^{\text {cd }} \\
(3.38)\end{array}$ & $\begin{array}{l}2.02^{\mathrm{e}} \\
(3.58)\end{array}$ & $\begin{array}{l}2.13^{\mathrm{d}} \\
(4.04)\end{array}$ & $\begin{array}{l}2.08^{\mathrm{e}} \\
(3.83)\end{array}$ & $\begin{array}{l}2.13^{\mathrm{e}} \\
(4.04)\end{array}$ & $\begin{array}{l}2.25^{\mathrm{d}} \\
(4.56)\end{array}$ & $\begin{array}{l}2.19^{\mathrm{e}} \\
(4.30)\end{array}$ & $\begin{array}{l}2.08^{\mathrm{e}} \\
(3.83)\end{array}$ \\
\hline 10 & Control & $\begin{array}{l}1.75 \\
(2.56)\end{array}$ & $\begin{array}{l}2.36^{\mathrm{e}} \\
(5.07)\end{array}$ & $\begin{array}{l}2.51^{\mathrm{e}} \\
(5.80)\end{array}$ & $\begin{array}{l}2.44^{\mathrm{e}} \\
(5.45)\end{array}$ & $\begin{array}{c}2.66^{f} \\
(6.58)\end{array}$ & $\begin{array}{l}2.77^{\mathrm{e}} \\
(7.17)\end{array}$ & $\begin{array}{c}2.72^{f} \\
(6.90)\end{array}$ & $\begin{array}{c}2.88^{f} \\
(7.79)\end{array}$ & $\begin{array}{l}3.00^{\mathrm{e}} \\
(8.50)\end{array}$ & $\begin{array}{c}2.95^{\mathrm{f}} \\
(8.20)\end{array}$ & $\begin{array}{l}2.70^{f} \\
(6.79)\end{array}$ \\
\hline \multicolumn{2}{|c|}{ S. Em. \pm Treatment $(T)$} & 0.06 & 0.06 & 0.06 & 0.05 & 0.07 & 0.05 & 0.05 & 0.05 & 0.07 & 0.05 & 0.03 \\
\hline & Location (L) & 0.03 & 0.04 & 0.03 & 0.03 & 0.04 & 0.03 & 0.03 & 0.03 & 0.04 & 0.03 & 0.02 \\
\hline & $\mathbf{T} \times \mathbf{L}$ & 0.11 & 0.12 & 0.12 & 0.09 & 0.14 & 0.11 & 0.09 & 0.11 & 0.14 & 0.09 & 0.05 \\
\hline \multicolumn{2}{|c|}{ C.D. at $0.05 \%$} & NS & $\mathrm{S}$ & S & $\mathrm{S}$ & $\mathrm{S}$ & $\mathrm{S}$ & $\mathrm{S}$ & $\mathrm{S}$ & $\mathrm{S}$ & $\mathrm{S}$ & $\mathrm{S}$ \\
\hline \multicolumn{2}{|r|}{ C. V. \% } & 10.04 & 12.25 & 10.75 & 11.24 & 13.31 & 10.43 & 11.81 & 10.64 & 12.81 & 11.81 & 11.79 \\
\hline
\end{tabular}


Table2 Evaluation of bio-pesticides against plant damage caused by fall armyworm, S. frugiperda infesting maize (Pooled over periods, sprays and locations)

\begin{tabular}{|c|c|c|c|c|c|c|c|c|c|c|c|c|}
\hline \multirow{3}{*}{$\begin{array}{l}\text { Tr. } \\
\text { No. }\end{array}$} & \multirow[t]{3}{*}{ Treatments } & \multicolumn{11}{|c|}{ Plant damage (\%) days after spray } \\
\hline & & \multirow{2}{*}{$\begin{array}{l}\text { Before } \\
\text { spray }\end{array}$} & \multicolumn{3}{|c|}{ First } & \multicolumn{3}{|c|}{ Second } & \multicolumn{3}{|c|}{ Third } & \multirow{2}{*}{$\begin{array}{l}\text { Pooled over } \\
\text { periods and } \\
\text { sprays }\end{array}$} \\
\hline & & & 5 & 10 & Pooled & 5 & 10 & Pooled & 5 & 10 & Pooled & \\
\hline 1 & $\begin{array}{l}\text { Beauveria bassiana } 5 \% \mathrm{WP} \\
(40 \mathrm{~g} / 10 \text { lit water) }\end{array}$ & $\begin{array}{l}32.25 \\
(28.47)\end{array}$ & $\begin{array}{l}27.63^{\mathrm{abc}} \\
(21.51)\end{array}$ & $\begin{array}{l}32.22^{\text {cd }} \\
(28.43)\end{array}$ & $\begin{array}{l}29.93^{\mathrm{b}} \\
(24.89)\end{array}$ & $\begin{array}{l}29.03^{\text {bcd }} \\
(23.55)\end{array}$ & $\begin{array}{l}28.53^{b c} \\
(22.81)\end{array}$ & $\begin{array}{l}28.78^{b} \\
(23.18)\end{array}$ & $\begin{array}{l}25.08^{b c} \\
(17.97)\end{array}$ & $\begin{array}{l}28.37^{b} \\
(22.58)\end{array}$ & $\begin{array}{l}26.72^{b} \\
(20.22)\end{array}$ & $\begin{array}{l}28.48^{\mathrm{b}} \\
(22.74)\end{array}$ \\
\hline 2 & $\begin{array}{l}\text { Metarhizium anisopliae } 1.15 \% \mathrm{WP} \\
\text { (40 g/10 lit water) }\end{array}$ & $\begin{array}{c}30.01 \\
(25.02)\end{array}$ & $\begin{array}{l}29.20^{\mathrm{bc}} \\
(23.80)\end{array}$ & $\begin{array}{l}35.10^{\text {cde }} \\
(33.06)\end{array}$ & $\begin{array}{l}32.15^{\mathrm{bc}} \\
(28.32)\end{array}$ & $\begin{array}{l}31.32^{\text {cd }} \\
(27.02)\end{array}$ & $\begin{array}{l}30.84^{c} \\
(26.41)\end{array}$ & $\begin{array}{l}31.08^{\mathrm{b}} \\
(26.65)\end{array}$ & $\begin{array}{l}28.53^{\text {cd }} \\
(22.81)\end{array}$ & $\begin{array}{l}30.84^{\mathrm{bc}} \\
(26.28)\end{array}$ & $\begin{array}{l}29.68^{\mathrm{b}} \\
(24.52)\end{array}$ & $\begin{array}{l}30.97^{b c} \\
(26.48)\end{array}$ \\
\hline 3 & $\begin{array}{l}\text { Bacillus thuringiensis } 1 \% \mathrm{WG} \\
\text { (20 g/10 lit water) }\end{array}$ & $\begin{array}{l}32.98 \\
(29.63)\end{array}$ & $\begin{array}{l}25.48^{\mathrm{ab}} \\
(18.51)\end{array}$ & $\begin{array}{l}28.03^{a b} \\
(22.08)\end{array}$ & $\begin{array}{l}26.76^{\mathrm{a}} \\
(20.27)\end{array}$ & $\begin{array}{l}24.58^{\mathrm{ab}} \\
(17.30)\end{array}$ & $\begin{array}{l}25.48^{\mathrm{ab}} \\
(18.51)\end{array}$ & $\begin{array}{l}25.03^{\mathrm{a}} \\
(17.90)\end{array}$ & $\begin{array}{l}22.03^{\mathrm{ab}} \\
(14.07)\end{array}$ & $\begin{array}{l}23.68^{\mathrm{a}} \\
(16.13)\end{array}$ & $\begin{array}{l}22.86^{\mathrm{a}} \\
(15.09)\end{array}$ & $\begin{array}{l}24.88^{\mathrm{a}} \\
(17.70)\end{array}$ \\
\hline 4 & $\begin{array}{l}\text { Nomuraea rileyi } 1 \% \mathrm{WP} \\
(40 \mathrm{~g} / 10 \text { lit water })\end{array}$ & $\begin{array}{c}32.32 \\
(28.58)\end{array}$ & $\begin{array}{l}23.68^{\mathrm{a}} \\
(16.13)\end{array}$ & $\begin{array}{l}27.12^{\mathrm{a}} \\
(20.78)\end{array}$ & $\begin{array}{l}25.40^{\mathrm{a}} \\
(18.40)\end{array}$ & $\begin{array}{l}23.68^{\mathrm{a}} \\
(16.13)\end{array}$ & $\begin{array}{l}22.94^{\mathrm{a}} \\
(15.19)\end{array}$ & $\begin{array}{l}23.31^{\mathrm{a}} \\
(15.66)\end{array}$ & $\begin{array}{l}18.18^{\mathrm{a}} \\
(9.73)\end{array}$ & $\begin{array}{l}22.77^{\mathrm{a}} \\
(14.98)\end{array}$ & $\begin{array}{l}20.48^{\mathrm{a}} \\
(12.24)\end{array}$ & $\begin{array}{l}23.06^{\mathrm{a}} \\
(15.34)\end{array}$ \\
\hline 5 & $\begin{array}{l}\text { Azadirachtin } 1500 \mathrm{ppm} \\
(40 \mathrm{ml} / 10 \text { lit water) }\end{array}$ & $\begin{array}{l}30.84 \\
(26.28)\end{array}$ & $\begin{array}{l}28.53^{\mathrm{bc}} \\
(22.81)\end{array}$ & $\begin{array}{l}31.58^{b c} \\
(27.42)\end{array}$ & $\begin{array}{l}30.06^{\mathrm{b}} \\
(25.09)\end{array}$ & $\begin{array}{l}28.53^{\mathrm{bc}} \\
(22.81)\end{array}$ & $\begin{array}{l}28.53^{b c} \\
(22.81)\end{array}$ & $\begin{array}{l}28.54^{\mathrm{b}} \\
(22.83)\end{array}$ & $\begin{array}{l}26.72^{c} \\
(20.22)\end{array}$ & $\begin{array}{l}29.94^{b} \\
(24.91)\end{array}$ & $\begin{array}{l}28.33^{\mathrm{b}} \\
(22.52)\end{array}$ & $\begin{array}{l}28.97^{\mathrm{b}} \\
(23.46)\end{array}$ \\
\hline 6 & $\begin{array}{l}\text { Neem seed kernel extract } 5 \% \\
(500 \mathrm{~g} / 10 \text { lit water) }\end{array}$ & $\begin{array}{c}31.34 \\
(27.05)\end{array}$ & $\begin{array}{l}28.37^{\mathrm{bc}} \\
(22.58)\end{array}$ & $\begin{array}{l}33.63^{\text {cd }} \\
(30.67)\end{array}$ & $\begin{array}{l}30.99^{b} \\
(26.51)\end{array}$ & $\begin{array}{l}30.67^{\mathrm{cd}} \\
(26.02)\end{array}$ & $\begin{array}{l}31.58^{\text {cd }} \\
(27.42)\end{array}$ & $\begin{array}{l}31.13^{\mathrm{b}} \\
(26.73)\end{array}$ & $\begin{array}{l}28.53^{\text {cd }} \\
(22.81)\end{array}$ & $\begin{array}{l}30.84^{\mathrm{bc}} \\
(26.28)\end{array}$ & $\begin{array}{l}29.68^{\mathrm{b}} \\
(24.52)\end{array}$ & $\begin{array}{l}30.61^{\mathrm{bc}} \\
(25.93)\end{array}$ \\
\hline 7 & $\begin{array}{l}\text { Tobacco decoction } 2 \% \text { (cold method) } \\
\text { (200 g/10 lit water) }\end{array}$ & $\begin{array}{l}30.01 \\
(25.02)\end{array}$ & $\begin{array}{l}29.27^{b c} \\
(23.90)\end{array}$ & $\begin{array}{l}35.84^{\text {de }} \\
(34.28)\end{array}$ & $\begin{array}{l}32.55^{\mathrm{bc}} \\
(28.95)\end{array}$ & $\begin{array}{l}33.69^{\mathrm{de}} \\
(30.77)\end{array}$ & $\begin{array}{l}35.84^{\mathrm{de}} \\
(34.28)\end{array}$ & $\begin{array}{l}34.77^{\mathrm{c}} \\
(32.52)\end{array}$ & $\begin{array}{l}32.96^{\mathrm{de}} \\
(29.60)\end{array}$ & $\begin{array}{l}35.10^{\text {cd }} \\
(33.06)\end{array}$ & $\begin{array}{l}34.03^{\mathrm{c}} \\
(31.32)\end{array}$ & $\begin{array}{l}33.79^{\text {cd }} \\
(30.93)\end{array}$ \\
\hline 8 & $\begin{array}{l}\text { Lantana camara leaf water extract } 10 \% \\
(1000 \mathrm{~g} / 10 \text { lit water) }\end{array}$ & $\begin{array}{c}30.67 \\
(26.02)\end{array}$ & $\begin{array}{l}30.01^{\mathrm{c}} \\
(25.02)\end{array}$ & $\begin{array}{l}38.36^{\mathrm{e}} \\
(38.51)\end{array}$ & $\begin{array}{l}34.19^{c} \\
(32.58)\end{array}$ & $\begin{array}{l}37.05^{\mathrm{e}} \\
(36.30)\end{array}$ & $\begin{array}{l}38.46^{\mathrm{ef}} \\
(38.68)\end{array}$ & $\begin{array}{l}37.76^{\mathrm{cd}} \\
(37.50)\end{array}$ & $\begin{array}{l}35.00^{\text {ef }} \\
(32.90)\end{array}$ & $\begin{array}{l}37.05^{\text {de }} \\
(36.30)\end{array}$ & $\begin{array}{l}36.03^{\mathrm{c}} \\
(34.60)\end{array}$ & $\begin{array}{l}35.99^{\mathrm{de}} \\
(34.53)\end{array}$ \\
\hline 9 & $\begin{array}{l}\text { Green chilli water extract } 10 \% \\
(1000 \mathrm{~g} / 10 \text { lit water) }\end{array}$ & $\begin{array}{l}32.98 \\
(29.63)\end{array}$ & $\begin{array}{l}31.58^{\mathrm{c}} \\
(27.42)\end{array}$ & $\begin{array}{l}38.39^{\mathrm{e}} \\
(38.57)\end{array}$ & $\begin{array}{l}34.98^{\mathrm{c}} \\
(32.87)\end{array}$ & $\begin{array}{l}37.72^{\mathrm{e}} \\
(37.43)\end{array}$ & $\begin{array}{l}40.44^{f} \\
(42.07)\end{array}$ & $\begin{array}{l}39.08^{\mathrm{d}} \\
(39.74)\end{array}$ & $\begin{array}{l}38.36^{\mathrm{f}} \\
(38.51)\end{array}$ & $\begin{array}{l}41.08^{\mathrm{e}} \\
(43.18)\end{array}$ & $\begin{array}{l}39.72^{\mathrm{d}} \\
(40.84)\end{array}$ & $\begin{array}{l}37.93^{\mathrm{e}} \\
(37.79)\end{array}$ \\
\hline 10 & Control & $\begin{array}{l}28.37 \\
(22.58)\end{array}$ & $\begin{array}{l}40.44^{\mathrm{d}} \\
(42.07)\end{array}$ & $\begin{array}{l}46.93^{f} \\
(53.37)\end{array}$ & $\begin{array}{l}43.78^{\mathrm{d}} \\
(47.87)\end{array}$ & $\begin{array}{c}50.28^{f} \\
(59.16)\end{array}$ & $\begin{array}{l}55.02^{\mathrm{g}} \\
(67.13)\end{array}$ & $\begin{array}{l}52.66^{\mathrm{e}} \\
(63.21)\end{array}$ & $\begin{array}{l}59.35^{\mathrm{g}} \\
(74.01)\end{array}$ & $\begin{array}{l}65.38^{f} \\
(82.64)\end{array}$ & $\begin{array}{l}62.36^{\mathrm{e}} \\
(78.48)\end{array}$ & $\begin{array}{l}52.90^{f} \\
(63.61)\end{array}$ \\
\hline \multicolumn{2}{|c|}{ S. Em. \pm Treatment (T) } & 1.39 & 1.37 & 1.27 & 0.98 & 1.45 & 1.34 & 1.02 & 1.49 & 1.40 & 1.04 & 1.08 \\
\hline & Location (L) & 0.83 & 0.79 & 0.70 & 0.54 & 0.69 & 0.78 & 0.56 & 0.85 & 0.78 & 0.57 & 0.32 \\
\hline & T X L & 2.64 & 2.52 & 2.21 & 1.69 & 2.51 & 2.47 & 1.77 & 2.68 & 2.46 & 1.80 & 1.01 \\
\hline \multicolumn{2}{|c|}{ C.D. at $0.05 \%$} & NS & $\mathrm{S}$ & $\mathrm{S}$ & $\mathrm{S}$ & $\mathrm{S}$ & $\mathrm{S}$ & $\mathrm{S}$ & $\mathrm{S}$ & $\mathrm{S}$ & $\mathrm{S}$ & $\mathrm{S}$ \\
\hline \multicolumn{2}{|c|}{ C. V. \% } & 14.67 & 14.84 & 11.05 & 12.96 & 13.34 & 12.70 & 13.09 & 14.78 & 12.39 & 13.40 & 13.80 \\
\hline
\end{tabular}


Table.3 Evaluation of bio-pesticide against fall armyworm, S. frugiperda and its plant damage in maize (Pooled over locations)

\begin{tabular}{|c|c|c|c|c|c|c|c|c|c|}
\hline \multirow{2}{*}{$\begin{array}{l}\text { Tr. } \\
\text { No. }\end{array}$} & \multirow[t]{2}{*}{ Treatment } & \multicolumn{4}{|c|}{ No. of larva(e)/10 plants } & \multicolumn{4}{|c|}{ Plant damage $(\%)$} \\
\hline & & Anand & Sansoli & Godhra & Pooled & Anand & Sansoli & Godhra & Pooled \\
\hline 1 & $\begin{array}{l}\text { Beauveria bassiana } 5 \% \mathrm{WP} \\
\text { (40 g/10 lit water) }\end{array}$ & $\begin{array}{c}1.99 \\
(3.46)^{*}\end{array}$ & $\begin{array}{l}1.63^{\mathrm{bc}} \\
(2.16)\end{array}$ & $\begin{array}{l}1.52^{\mathrm{ab}} \\
(1.81)\end{array}$ & $\begin{array}{l}1.71^{\mathrm{b}} \\
(2.42)\end{array}$ & $\begin{array}{c}35.82^{\mathrm{b}} \\
(34.25)^{* *}\end{array}$ & $\begin{array}{l}26.76^{\mathrm{cd}} \\
(20.27)\end{array}$ & $\begin{array}{l}22.86^{\mathrm{bc}} \\
(15.09)\end{array}$ & $\begin{array}{l}28.48^{b} \\
(22.74)\end{array}$ \\
\hline 2 & $\begin{array}{l}\text { Metarhizium anisopliae } 1.15 \% \text { WP } \\
\text { (40 g/10 lit water) }\end{array}$ & $\begin{array}{c}2.06 \\
(3.74)\end{array}$ & $\begin{array}{l}1.72^{\text {cd }} \\
(2.46)\end{array}$ & $\begin{array}{l}1.64^{\mathrm{b}} \\
(2.19)\end{array}$ & $\begin{array}{l}1.81^{\mathrm{c}} \\
(2.78)\end{array}$ & $\begin{array}{l}37.85^{\mathrm{bc}} \\
(37.65)\end{array}$ & $\begin{array}{l}28.69^{\mathrm{de}} \\
(23.05)\end{array}$ & $\begin{array}{l}26.39^{\text {de }} \\
(19.76)\end{array}$ & $\begin{array}{l}30.97^{b c} \\
(26.48)\end{array}$ \\
\hline 3 & $\begin{array}{l}\text { Bacillus thuringiensis } 1 \% \mathrm{WG} \\
\text { (20 g/10 lit water) }\end{array}$ & $\begin{array}{c}1.82 \\
(2.81)\end{array}$ & $\begin{array}{l}1.44^{\mathrm{a}} \\
(1.57)\end{array}$ & $\begin{array}{l}1.49^{\mathrm{ab}} \\
(1.72)\end{array}$ & $\begin{array}{l}1.59^{\mathrm{a}} \\
(2.03)\end{array}$ & $\begin{array}{l}25.31^{\mathrm{a}} \\
(18.28)\end{array}$ & $\begin{array}{l}23.39^{\mathrm{ab}} \\
(15.76)\end{array}$ & $\begin{array}{l}22.94 \mathrm{~cd} \\
(15.19)\end{array}$ & $\begin{array}{r}24.88^{a} \\
(17.70)\end{array}$ \\
\hline 4 & $\begin{array}{l}\text { Nomuraea rileyi } 1 \% \mathrm{WP} \\
(40 \mathrm{~g} / 10 \text { lit water) }\end{array}$ & $\begin{array}{l}1.78 \\
(2.67)\end{array}$ & $\begin{array}{l}1.40^{\mathrm{a}} \\
(1.46)\end{array}$ & $1.36^{\mathrm{a}}(1.35)$ & $\begin{array}{l}1.52^{\mathrm{a}} \\
(1.81)\end{array}$ & $\begin{array}{l}27.49^{\mathrm{a}} \\
(21.31)\end{array}$ & $\begin{array}{l}22.04^{\mathrm{a}} \\
(14.08)\end{array}$ & $\begin{array}{l}19.66^{\mathrm{a}} \\
(11.32)\end{array}$ & $\begin{array}{l}23.06^{\mathrm{a}} \\
(15.34)\end{array}$ \\
\hline 5 & $\begin{array}{l}\text { Azadirachtin } 1500 \text { ppm } \\
(40 \mathrm{ml} / 10 \text { lit water })\end{array}$ & $\begin{array}{c}2.00 \\
(3.50)\end{array}$ & $\begin{array}{l}1.56^{\mathrm{b}} \\
(1.93)\end{array}$ & $1.59^{\mathrm{b}}(2.03)$ & $\begin{array}{l}1.72^{\mathrm{bc}} \\
(2.46)\end{array}$ & $\begin{array}{l}35.87^{\mathrm{b}} \\
(37.33)\end{array}$ & $\begin{array}{l}24.75^{\mathrm{bc}} \\
(17.53)\end{array}$ & $\begin{array}{l}26.30^{\mathrm{de}} \\
(19.63)\end{array}$ & $\begin{array}{l}28.97^{b} \\
(23.46)\end{array}$ \\
\hline 6 & $\begin{array}{l}\text { Neem seed kernel extract } 5 \% \\
(500 \mathrm{~g} / 10 \text { lit water })\end{array}$ & $\begin{array}{c}2.01 \\
(3.54)\end{array}$ & $\begin{array}{l}1.61^{\mathrm{bc}} \\
(2.09)\end{array}$ & $\begin{array}{l}1.67^{\mathrm{bc}} \\
(2.29)\end{array}$ & $\begin{array}{l}1.77^{\mathrm{bc}} \\
(2.63)\end{array}$ & $\begin{array}{l}36.86^{\mathrm{bc}} \\
(35.98)\end{array}$ & $\begin{array}{l}26.02^{\mathrm{cd}} \\
(19.24)\end{array}$ & $\begin{array}{l}28.93^{\text {ef }} \\
(23.40)\end{array}$ & $\begin{array}{l}30.61^{b c} \\
(25.93)\end{array}$ \\
\hline 7 & $\begin{array}{l}\text { Tobacco decoction } 2 \% \text { (cold method) } \\
\text { (200 g/10 lit water) }\end{array}$ & $\begin{array}{c}2.15 \\
(4.12)\end{array}$ & $\begin{array}{l}1.82^{\mathrm{de}} \\
(2.81)\end{array}$ & $\begin{array}{l}1.84^{\mathrm{cd}} \\
(2.89)\end{array}$ & $\begin{array}{l}1.94^{\mathrm{d}} \\
(3.26)\end{array}$ & $\begin{array}{l}39.48^{c} \\
(40.43)\end{array}$ & $\begin{array}{l}30.90^{\mathrm{ef}} \\
(26.37)\end{array}$ & $\begin{array}{l}30.98^{f g} \\
(26.50)\end{array}$ & $\begin{array}{l}33.79^{\mathrm{cd}} \\
(30.93)\end{array}$ \\
\hline 8 & $\begin{array}{l}\text { Lantana camara leaf water extract } \\
10 \%(1000 \mathrm{~g} / 10 \text { lit water })\end{array}$ & $\begin{array}{c}2.23 \\
(4.47)\end{array}$ & $\begin{array}{l}1.91^{\mathrm{ef}} \\
(3.15)\end{array}$ & $1.96^{\mathrm{d}}(3.34)$ & $\begin{array}{l}2.04^{\mathrm{e}} \\
(3.66)\end{array}$ & $\begin{array}{l}43.03^{d} \\
(46.56)\end{array}$ & $\begin{array}{l}32.98 f^{g} \\
(29.63)\end{array}$ & $\begin{array}{l}31.97^{\mathrm{fg}} \\
(28.03)\end{array}$ & $\begin{array}{l}35.99^{\mathrm{de}} \\
(34.53)\end{array}$ \\
\hline 9 & $\begin{array}{l}\text { Green chilli water extract } 10 \% \\
(1000 \mathrm{~g} / 10 \text { lit water) }\end{array}$ & $\begin{array}{c}2.27 \\
(4.65)\end{array}$ & $\begin{array}{l}1.96^{\mathrm{f}} \\
(3.34)\end{array}$ & $2.01^{\mathrm{d}}(3.54)$ & $\begin{array}{l}2.08^{\mathrm{e}} \\
(3.83)\end{array}$ & $\begin{array}{l}44.96^{d} \\
(49.93)\end{array}$ & $\begin{array}{c}35.10^{\mathrm{g}} \\
(33.06)\end{array}$ & $\begin{array}{c}33.72^{\mathrm{g}} \\
(30.82)\end{array}$ & $\begin{array}{c}37.93^{\mathrm{e}} \\
(37.79)\end{array}$ \\
\hline 10 & Control & $\begin{array}{c}2.92 \\
(8.03)\end{array}$ & $\begin{array}{l}2.56^{\mathrm{g}} \\
(6.05)\end{array}$ & $\begin{array}{l}2.61^{\mathrm{e}} \\
(6.31)\end{array}$ & $\begin{array}{l}2.70^{f} \\
(6.79)\end{array}$ & $\begin{array}{l}56.14^{\mathrm{e}} \\
(68.96)\end{array}$ & $\begin{array}{l}49.04^{\mathrm{h}} \\
(57.03)\end{array}$ & $\begin{array}{l}53.52^{\mathrm{h}} \\
(64.65)\end{array}$ & $\begin{array}{c}52.90^{f} \\
(63.61)\end{array}$ \\
\hline & S. Em. \pm Treatment $(T)$ & 0.05 & 0.04 & 0.05 & 0.03 & 1.05 & 0.83 & 1.11 & 1.08 \\
\hline & Location $(\mathrm{L})$ & - & - & - & 0.02 & - & - & - & 0.32 \\
\hline & $\mathbf{T} \times \mathbf{L}$ & - & - & - & 0.05 & - & - & - & 1.01 \\
\hline C. V. & & 11.83 & 9.27 & 13.64 & 11.76 & 11.63 & 11.76 & 15.91 & 13.80 \\
\hline \multirow[t]{2}{*}{ Note: } & \multicolumn{9}{|c|}{ 1. *Figures in parenthesis are retransformed values; those outside are $\sqrt{x+0.5}$ transformed values } \\
\hline & \multicolumn{9}{|c|}{$\begin{array}{l}\text { 2.**Figures in parenthesis are retransformed values; those outside are arc sine transformed values } \\
\text { 3. Treatment mean(s) with the letter(s) in common are not significant by DNMRT at } 5 \% \text { level of significance } \\
\text { 4. Significant parameters and its interactions: T, P, L, T } \mathrm{S}, \mathrm{P} \times \mathrm{S} \text {, where } \mathrm{P}=\mathrm{Period} \text { and } \mathrm{S}=\text { Spray and } \\
\text { 5. Significant parameters and its interactions: T, P, L, T } \mathrm{S}, \mathrm{P} \times \mathrm{S}, \mathrm{T} \times \mathrm{L} \text { (For plant damage) }\end{array}$} \\
\hline
\end{tabular}


Table.4 Evaluation of bio-pesticides against cob damage caused by fall armyworm, S. frugiperda in maize

\begin{tabular}{|c|c|c|c|c|c|}
\hline \multirow[t]{2}{*}{ Tr. No. } & \multirow[t]{2}{*}{ Treatments } & \multicolumn{4}{|c|}{ Cob damage (\%) } \\
\hline & & Anand & Sansoli & Godhra & Pooled \\
\hline 1 & $\begin{array}{l}\text { Beauveria bassiana } 5 \% \mathrm{WP} \\
\text { (40 g/10 lit water) }\end{array}$ & $\begin{array}{l}32.98^{\mathrm{b}} \\
(29.63)\end{array}$ & $\begin{array}{l}32.99^{\mathrm{b}} \\
(29.65)\end{array}$ & $\begin{array}{l}30.98^{\mathrm{b}} \\
(26.50)\end{array}$ & $\begin{array}{l}32.32^{\mathrm{b}} \\
(28.58)\end{array}$ \\
\hline 2 & $\begin{array}{l}\text { Metarhizium anisopliae } 1.15 \% \mathrm{WP} \\
\text { (40 g/10 lit water) }\end{array}$ & $\begin{array}{r}35.20^{\mathrm{b}} \\
(33.23)\end{array}$ & $\begin{array}{l}35.20^{\mathrm{b}} \\
(33.23)\end{array}$ & $\begin{array}{l}35.20^{\mathrm{b}} \\
(33.23)\end{array}$ & $\begin{array}{l}35.20^{\mathrm{b}} \\
(33.23)\end{array}$ \\
\hline 3 & $\begin{array}{l}\text { Bacillus thuringiensis } 1 \% \mathrm{WG} \\
(20 \mathrm{~g} / 10 \text { lit water) }\end{array}$ & $\begin{aligned} 23.84^{\mathrm{a}} \\
(16.34)\end{aligned}$ & $\begin{aligned} 23.84^{\mathrm{a}} \\
(16.34)\end{aligned}$ & $\begin{array}{r}21.13^{\mathrm{a}} \\
(12.99)\end{array}$ & $\begin{array}{r}22.94^{\mathrm{a}} \\
(15.19)\end{array}$ \\
\hline 4 & $\begin{array}{l}\text { Nomuraea rileyi } 1 \% \mathrm{WP} \\
(40 \mathrm{~g} / 10 \text { lit water) }\end{array}$ & $\begin{array}{r}23.84^{\mathrm{a}} \\
(16.34)\end{array}$ & $\begin{array}{r}23.84^{\mathrm{a}} \\
(16.34)\end{array}$ & $\begin{array}{r}21.13^{\mathrm{a}} \\
(12.99)\end{array}$ & $\begin{array}{l}22.94^{\mathrm{a}} \\
(15.19)\end{array}$ \\
\hline 5 & $\begin{array}{l}\text { Azadirachtin } 1500 \mathrm{ppm} \\
\text { (40 ml/10 lit water) }\end{array}$ & $\begin{array}{l}32.98^{\mathrm{b}} \\
(29.63)\end{array}$ & $\begin{array}{l}32.99^{b} \\
(29.65)\end{array}$ & $\begin{array}{l}30.98^{\mathrm{b}} \\
(26.50)\end{array}$ & $\begin{array}{l}32.32^{b} \\
(28.58)\end{array}$ \\
\hline 6 & $\begin{array}{l}\text { Neem seed kernel extract } 5 \% \\
(500 \mathrm{~g} / 10 \text { lit water) }\end{array}$ & $\begin{array}{l}35.20^{\mathrm{b}} \\
(33.23)\end{array}$ & $\begin{array}{l}35.20^{\mathrm{b}} \\
(33.23)\end{array}$ & $\begin{array}{l}35.20^{\mathrm{b}} \\
(33.23)\end{array}$ & $\begin{array}{l}35.20^{b} \\
(33.23)\end{array}$ \\
\hline 7 & $\begin{array}{l}\text { Tobacco decoction } 2 \% \text { (cold method) } \\
\text { (200 g/10 lit water) }\end{array}$ & $\begin{array}{l}35.20^{\mathrm{b}} \\
(33.23)\end{array}$ & $\begin{array}{l}35.20^{\mathrm{b}} \\
(33.23)\end{array}$ & $\begin{array}{l}35.20^{\mathrm{b}} \\
(33.23)\end{array}$ & $\begin{array}{l}35.20^{\mathrm{b}} \\
(33.23)\end{array}$ \\
\hline 8 & $\begin{array}{l}\text { Lantana camara leaf water extract } 10 \%(1000 \mathrm{~g} / 10 \\
\text { lit water) }\end{array}$ & $\begin{array}{l}37.21^{\mathrm{b}} \\
(36.57)\end{array}$ & $\begin{array}{l}37.21^{\mathrm{b}} \\
(36.57)\end{array}$ & $\begin{array}{l}35.20^{\mathrm{b}} \\
(33.23)\end{array}$ & $\begin{array}{l}36.54^{b} \\
(35.45)\end{array}$ \\
\hline 9 & $\begin{array}{l}\text { Green chilli water extract } 10 \% \\
(1000 \mathrm{~g} / 10 \text { lit water) }\end{array}$ & $\begin{array}{l}37.21^{\mathrm{b}} \\
(36.27)\end{array}$ & $\begin{array}{l}37.21^{\mathrm{b}} \\
(36.57)\end{array}$ & $\begin{array}{l}35.20^{\mathrm{b}} \\
(33.23)\end{array}$ & $\begin{array}{l}36.54^{\mathrm{b}} \\
(35.45)\end{array}$ \\
\hline 10 & Control & $\begin{array}{l}50.83^{\mathrm{c}} \\
(60.11)\end{array}$ & $\begin{array}{r}48.83^{\mathrm{c}} \\
(56.66)\end{array}$ & $\begin{array}{r}46.90^{\mathrm{c}} \\
(53.31)\end{array}$ & $\begin{array}{r}48.85^{\mathrm{c}} \\
(56.70)\end{array}$ \\
\hline \multicolumn{2}{|r|}{ Treatment $(\mathbf{T})$} & 2.82 & 2.67 & 2.31 & 1.32 \\
\hline & Location (L) & - & - & - & 0.82 \\
\hline \multirow{2}{*}{\multicolumn{2}{|c|}{$\mathbf{T} \times \mathbf{L}$}} & - & - & & 2.61 \\
\hline & & 14.20 & 13.48 & 12.25 & 13.37 \\
\hline \multicolumn{6}{|c|}{$\begin{array}{l}\text { Note: 1. Figures in parenthesis are retransformed values; those outside are arc sine transformed values } \\
\text { 2. Treatment mean(s) with the letter(s) in common are not significant by DNMRT at } 5 \% \text { level of significance } \\
\text { 3. Significant parameters and its interactions: T and L }\end{array}$} \\
\hline
\end{tabular}


Table.5 Effect of bio-pesticides on grain and fodder yield of maize

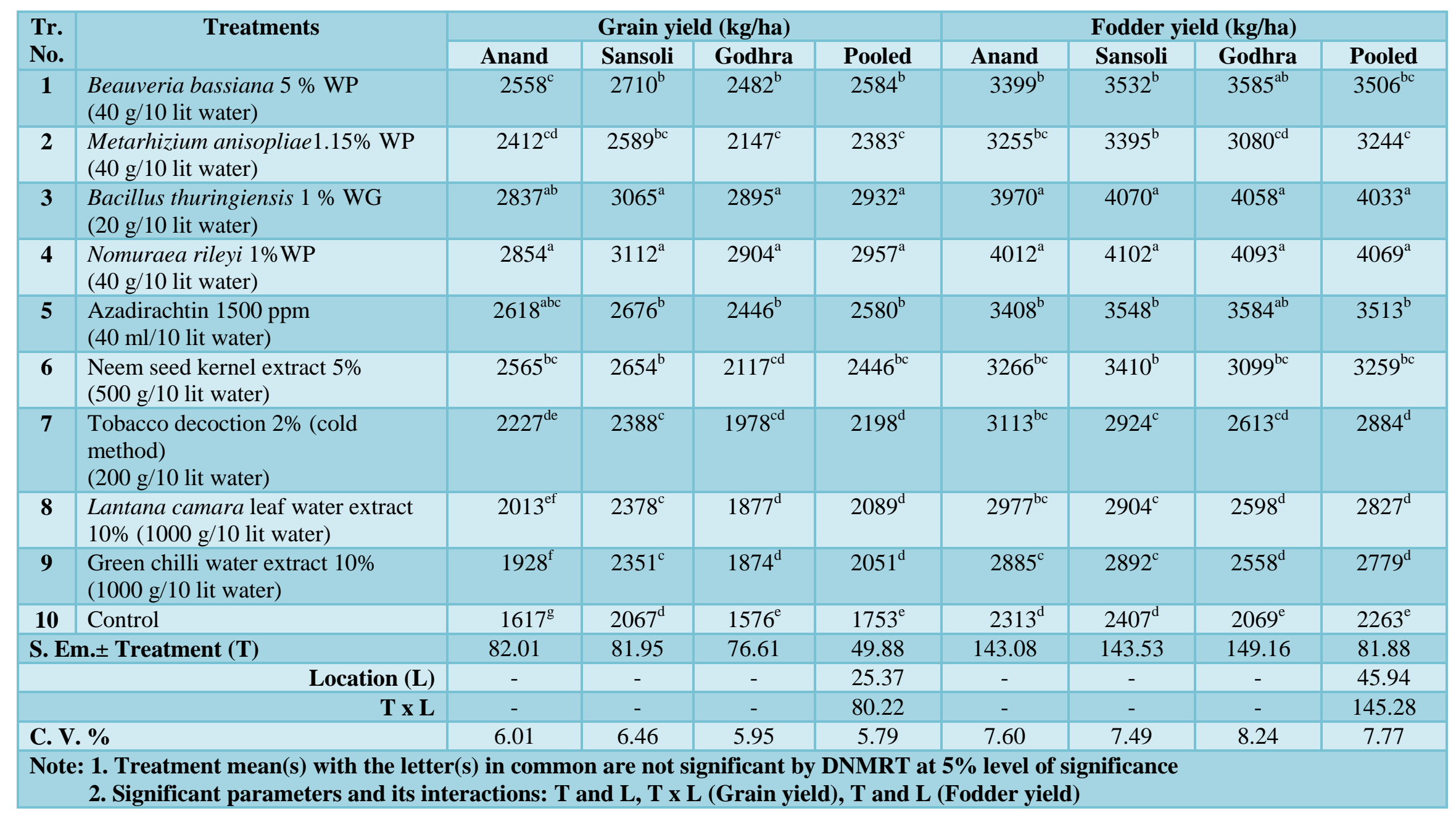


The data on pooled over periods of second spray differed significantly to each other. The significantly minimum plant damage was noticed in plots treated with $N$. rileyi $1 \%$ WP $(15.66 \%)$ and it was at par with $B$. thuringiensis $1 \%$ WG (17.90\%). These two treatments were found significantly superior to rest of the bio-pesticides. Azadirachtin $1500 \mathrm{ppm}$ (22.83\%), B. bassiana 5\% WP $(23.18 \%)$ and neem seed kernel extracts $(26.73 \%)$ were found equally effective in reducing the plant damage caused by fall armyworm. The green chilli water extract recorded maximum plant damage (39.74\%) and it was at par with L. camara leaf water extract $(37.50 \%)$. More or less similar results were found after pooled over periods of third spray as observed after pooled over periods of second spray.

Overall, the data on pooled over periods, sprays and locations showed minimum plant damage in the treatment of $N$. rileyi $1 \% \mathrm{WP}$ $(15.34 \%)$ and it was at par with $B$. thuringiensis $1 \% \mathrm{WG}(17.70 \%)$. B. bassiana $5 \% \mathrm{WP}(22.74 \%)$ and azadirachtin $1500 \mathrm{ppm}$ $(23.46 \%)$ were at par and next effective treatments against the pest. Of the tested biopesticides, green chilli water extract found least effective by recording the highest plant damage $(37.79 \%)$.

\section{Cob damage (\%)}

The data on cob damage pooled over the locations showed significant difference among the various biopesticides tested (Table 4). The lowest cob damage was recorded in plots treated with $N$. rileyi $1 \%$ WP $(15.19 \%)$ and $B$. thuringiensis (15.19\%). The treatments of $B$. bassiana 5\% WP and azadirachtin 1500 ppm found mediocre in their effectiveness against the pest. The highest cob damage recorded in green chilli water extract $(35.45$ $\%)$ and proved least effective in controlling the FAW in maize.

\section{Grain yield (Kg/ha)}

The highest grain yield recorded in the treatment of $N$. rileyi $1 \%$ WP $(2957 \mathrm{~kg} / \mathrm{ha})$ among all the treated biopesticides and it was at par with $B$. thuringiensis (2932 kg/ha). $B$. bassiana 5\% WP (2584 kg/ha), Azadirachtin $(2580 \mathrm{~kg} / \mathrm{ha})$ and neem seed kernel extract $(2446 \mathrm{~kg} / \mathrm{ha})$ were found equal in registering grain yield of maize. The green chilli water extract recorded the lowest grain yield (2051kg/ha) and it was at par with L. camara leaf water extract $(2089 \mathrm{~kg} / \mathrm{ha})$ and tobacco decoction $(2198 \mathrm{~kg} / \mathrm{ha})$.

\section{Fodder yield (Kg/ha)}

The highest fodder yield recorded in $N$. rileyi $1 \%$ WP (4069 kg/ha) and it was at par with $B$. thuringiensis $(4033 \mathrm{~kg} / \mathrm{ha})$. Azadirachtin 1500 ppm (3513.3 kg/ha), B. bassiana 5\% WP $(3506 \mathrm{~kg} / \mathrm{ha})$ and neem seed kernel extract $(3259 \mathrm{~kg} / \mathrm{ha})$ were found at par to each other in production of fodder. The green chilli water extract recorded the lowest (2779 $\mathrm{kg} / \mathrm{ha}$ ) fodder yield and it was at par with $L$. camara leaf water extract $(2827 \mathrm{~kg} / \mathrm{ha})$ and tobacco decoction $(2884 \mathrm{~kg} / \mathrm{ha})$.

These findings are in accordance with the findings of Mallapur et al., (2018) who reported the high potentiality of $N$. rileyi in combating the notorious invasive pest, $S$. frugiperda in maize, whereas in Andhra Pradesh 36.9 per cent infection of $N$. rileyi on $S$. litura in ground nut field was observed (Vimala, 1994 and Sridher, 1996). Unlike chemical approaches, the entomopathogenic fungi can self-perpetuate where in, the farmers will be provided with an added advantage of avoiding repeated spraying which would save time, labour and money as well it can safeguard the environment as it is an eco-friendly approach. According to Capalbo et al., (2001) mortality of neonate larvae was 100 per cent within two days of 
spraying of $B$. thuringiensis $(B t)$ and all larvae were found dead on leaves. Among the pathogens, B. thuringiensis, $M$. anisopliae and $B$. bassiana can cause significant mortality in FAW populations and help to reduce leaf defoliation in crops (Molina-Ochoa et al., 2003).

It can be concluded that $N$. rileyi 1\% WP (2 $\left.\mathrm{x} 10^{8} \mathrm{cfu} / \mathrm{g}\right) @ 40 \mathrm{~g} / 10$ litre water or $B$. thuringiensis var. kurstaki 1\% WG @ 20 g/10 litre water first at initiation of pest and subsequent two sprays at 10 days interval found effective and economical for the management of fall armyworm, $S$. frugiperda infesting maize.

\section{Acknowledgement}

We are grateful to Director of Research and Dean PG Studies, Principal and Dean (Agri.), BACA, Unit Head, ARS, AAU, Sansoli and Research Scientist, MMRS, AAU, Godhra for providing facilities for this research work.

\section{References}

Almeida de Moraes, A. R., Lourençao, A. L., Paterniani, A. M. E. G. Z. (2015). Resistance of conventional and isogenic transgenic maize hybrids to Spodoptera frugiperda (Lepidoptera: Noctuidae) Bragantia, Campinas, 74(1): 50-57.

Bohnenblust, E. W., Breining, J. A., Shaffer, J. A. Fleischer, S. J. Roth, G. W. and Tooker, J. F. (2014). Current European corn borer, Ostrinia nubilalis, injury levels in the north-eastern United States and the value of $B t$ field corn. Pest Management Science 70:1711-1719.

Capalbo D M Fontana, Fernando Hercule Valicente, Iracema de Oliveira Moraes and Lúcia Helena Pelizer (2001). Solidstate fermentation of Bacillus thuringiensis tolworthi to control fall armyworm in maize. EJB Electronic
Journal of Biotechnology. 4:(2): 1-4.

Capinera, J. (2008). Fall armyworm, Spodoptera frugiperda (J. E. Smith) (lepidoptera: noctuidae). Encyclopedia of Entomology, pp.1409-1412.

Cruz, L. Viana, P. A. and Waquil, J. M. (2002). Maize cultivation: vegetative and reproductive phase pests. Embrapa Maize and Sorghum Technical Report, 49.p. 8.

Mallapur CP, Anjan Kumar Naik, Sireesh Hagari, Praveen T, Patil RK and S Lingappa (2018). Potentiality of Nomuraea rileyi (Farlow) Samson against the fall armyworm, Spodoptera frugiperda (J E Smith) infesting maize. Journal of Entomology and Zoology Studies. 6(6): 1062-1067

Molina-Ochoa, J., Lezama-Gutierrez, R., Gonzalez- Ramirez, M., LopezEdwards, M., Rodriguez-Vega, M. A., \& Arceo-Palacios, F. (2003). Pathogens and parasitic nematodes associated with populations of fall armyworm (Lepidoptera: Noctuidae) larvae in Mexico. Florida Entomologist, 86, 244253.

Murúa, G. and Virla E. (2004). Population parameters of Spodoptera frugiperda (Smith) (Lep.:Noctuidae) fed on corn and two predominant grasses in Tucuman (Argentina). ActaZool Mex, 20:199-210.

Shylesha, A. N., Jalali, S. K., Gupta, A., Varshney, R., Venkatesan, T., Shetty, P., Ojha, R., Ganiger, P. C., Navik, O., Subahara, K., Bakthavatsalam, N., Ballal, C. R., and Raghavendra, A. (2018). Studies on new invasive pest Spodoptera frugiperda (J. E. Smith) (Lepidoptera: Noctuidae) and its natural enemies. J. of Biological control, 32:3.

Sisodiya, D. B., Raghnandan, B. L., Bhatt, N. A., Verma, H. S. Shewale, C. P., Timbdiya, B. G. and Borad, P. K. (2018). The fall armyworm, Spodoptera 
frugiperda (J. E. Smith) (Lepidoptera, Noctuidae) First report of new invasive pest in maize fields of Gujarat, India. Journal of Entomology and Zoology studies, 6(5): 2089-2091.

Sridher V, Prasad VO. (1996). Life table studies on natural population of Spodoptera litura on Ground nut. Annals of Plant Protection Sciences.
4:142-147.

Vimala DPS. (1994). Conidia production of the entomopathogenic fungus Nomuraea rileyi and its evaluation for control of Spodoptera litura (Fabr.) on Ricinus cummunis. $J$ Invertebrate Pathology.63:145-150.

\section{How to cite this article:}

Dhobi, C. B., M. B. Zala, H. S. Verma, D. B. Sisodiya, R. K. Thumar, M. B. Patel, J. K. Patel and Borad, P. K. 2020. Evaluation of Bio-pesticides against Fall Armyworm, Spodoptera frugiperda (J. E. Smith) in Maize. Int.J.Curr.Microbiol.App.Sci. 9(08): 1150-1160. doi: https://doi.org/10.20546/ijcmas.2020.908.127 